\title{
In-plane magnetic-field-induced Wigner crystallization in a two-electron quantum dot
}

\author{
B. Szafran, ${ }^{1,2}$ F. M. Peeters, ${ }^{1, *}$ S. Bednarek, ${ }^{2}$ and J. Adamowski ${ }^{2}$ \\ ${ }^{1}$ Departement Fysica, Universiteit Antwerpen, (Campus Drie Eiken), B-2610 Antwerpen, Belgium \\ ${ }^{2}$ Faculty of Physics and Nuclear Techniques, AGH University of Science and Technology, al. Mickiewicza 30, 30-059 Kraków, Poland
}

(Received 21 June 2004; revised manuscript received 31 August 2004; published 23 December 2004)

\begin{abstract}
The orbital effects of the in-plane magnetic field on a two-electron harmonic quantum dot are studied using a variational method. For flat quantum dots the singlet-triplet transitions appearing in a perpendicular magnetic field are absent in a magnetic field oriented parallel to the plane of confinement. Instead, a degeneracy of orbital energies for symmetric and antisymmetric states at high in-plane magnetic field is observed. This degeneracy is due to the formation of Wigner molecules in the laboratory frame of reference with charge islands elongated along the direction of the magnetic field and localized within the plane perpendicular to it.
\end{abstract}

DOI: 10.1103/PhysRevB.70.235335

PACS number(s): 73.21.-b, 73.22.Gk

\section{INTRODUCTION}

Epitaxially grown quantum dots ${ }^{1}$ usually have a flat geometry with a confinement in the growth direction much stronger than the in-plane confinement. Application of a magnetic field oriented along the growth direction leads to a number of extensively studied ${ }^{2}$ effects, i.e., angular momentum and spin transitions, which are observed in transport spectroscopy ${ }^{3}$ as cusps in the single-electron charging lines. A high perpendicular magnetic field induces separation of electron charges, i.e., Wigner crystallization, which for circular dots appears only in the internal degrees of freedom. Laboratory-frame Wigner crystallization is a realizable feature of the ground state only at the angular momentum transitions. ${ }^{5}$ On the other hand, Wigner molecules can be observed in anisotropic quantum dots if the system possesses a nondegenerate classical ${ }^{4}$ counterpart reproducing the symmetry of the confinement potential. ${ }^{5,6}$ The pinning of Wigner molecules by a local potential cavity 7,8 or by an external charged defect ${ }^{9}$ was studied recently. In this paper we consider the breaking of the rotational symmetry of the quantum dot by the application of an in-plane magnetic field, and we show that it can result in a laboratory-frame Wigner localization.

The effect of the external magnetic field on the electron system is proportional to its flux through the area encircled by the electrons. Therefore, the orbital effects of the in-plane magnetic field are weaker than those of the perpendicular field and in the limit of a strictly two-dimensional confinement the in-plane magnetic field does not influence the orbital wave functions. Such an in-plane magnetic field has been applied experimentally ${ }^{10-12}$ to investigate spin effects (Zeeman splitting and spin-orbit ${ }^{13,14}$ interactions). Nevertheless, the electron wave functions in real dots have a finite spread in the growth direction. In pillar quantum dots based on $\mathrm{Al}_{x} \mathrm{Ga}_{1-x} \mathrm{As} / \mathrm{In}_{x} \mathrm{Ga}_{1-x} \mathrm{As}$ double barrier heterostructures ${ }^{15}$ the quantum well has a width of $12 \mathrm{~nm}$, and the width of the GaAs quantum well in the planar vertical $\operatorname{dot}^{16}$ is about $17 \mathrm{~nm}$. The lateral quantum dots ${ }^{17}$ are based on a gated two-dimensional electron gas (2DEG) formed at a GaAs- $n \mathrm{Al}_{x} \mathrm{Ga}_{1-x} \mathrm{As}$ heterojunction. The vertical spread of the electron wave function in the 2DEG for typical values of the electron density and dopant concentration is also of the order $10-15 \mathrm{~nm} .{ }^{18}$ As a consequence, the orbital effects of the field are nonzero and can be visible for instance in the diamagnetic shifts ${ }^{12}$ of chemical potentials. The role of the in-plane magnetic field for the attenuation of tunneling between vertically ${ }^{19}$ and laterally ${ }^{20}$ coupled dots has been pointed out.

The effect of the in-plane magnetic field on few-electron systems in a single dot has not been studied so far. The purpose of the present paper is to provide such a study for the two-electron system-the simplest few-electron eigenproblem. We show that although for strong vertical confinement and relatively weak magnetic fields the orbital related triplet-singlet energy difference (the exchange energy ${ }^{20}$ ) is positive and approximately constant, its limit value in the high magnetic field falls to zero, which results from the separation of the electron charges appearing due to Wigner crystallization. In vertical and lateral quantum dots the confinement in the growth direction has a rectangular or triangular shape. In the present paper we are interested in the qualitative effects of the in-plane field, so we consider a harmonic confinement potential that largely simplifies the calculations due to the separation of the center-of-mass motion.

This paper is organized as follows. The next section outlines the theory and the method to solve the Hamiltonian eigenvalue problem. In Sec. III the results and discussion are given. The summary and conclusions are presented in Sec. IV.

\section{THEORY}

We consider a pair of electrons in a three-dimensional harmonic quantum dot, rotationally symmetric with respect to the $z$ axis and subject to a magnetic field oriented along the $x$ direction. We apply the Landau gauge $\mathbf{A}=(0,-B z, 0)$ under which the Hamiltonian reads

$$
\begin{aligned}
H= & -\hbar^{2}\left(\nabla_{1}^{2}+\nabla_{2}^{2}\right) / 2 m+m \omega^{2}\left(x_{1}^{2}+y_{1}^{2}+x_{2}^{2}+y_{2}^{2}\right) / 2 \\
& +m\left(\omega_{z}^{2}+\omega_{c}^{2}\right)\left(z_{1}^{2}+z_{2}^{2}\right) / 2+i \hbar \omega_{c}\left(z_{1} \partial / \partial y_{1}+z_{2} \partial / \partial y_{2}\right) \\
& +e^{2} / 4 \pi \epsilon \epsilon_{0}\left|\mathbf{r}_{1}-\mathbf{r}_{2}\right|,
\end{aligned}
$$

where $\hbar \omega_{z}$ is the confinement energy in the $z$ direction, $\hbar \omega$ is 
the energy of confinement in the $(x, y)$ plane, $m$ is the electron band mass, $\omega_{c}=e B / m$ is the cyclotron frequency, and $\epsilon$ is the dielectric constant. For our numerical calculations material data of GaAs are used $m=0.067$ and $\epsilon=12.9$. We study the orbital effects neglecting the spin Zeeman interaction, which, however, can be trivially accounted for.

Introducing center-of-mass $\mathbf{R}=\left(\mathbf{r}_{1}+\mathbf{r}_{2}\right) / 2$ and relative position $\mathbf{r}=\mathbf{r}_{1}-\mathbf{r}_{2}$ vectors, one can separate the Hamiltonian into a sum of center-of-mass and relative Hamiltonians $H$ $=H_{c m}+H_{r e l}$ with

$$
\begin{aligned}
H_{c m}= & -\hbar^{2} \nabla_{c m}^{2} / 2 M+M \omega^{2}\left(X^{2}+Y^{2}\right) / 2+M\left(\omega_{z}^{2}+\omega_{c}^{2}\right) Z^{2} / 2 \\
& +i \hbar \omega_{c} Z \partial / \partial Y
\end{aligned}
$$

and

$$
\begin{aligned}
H_{r e l}= & -\hbar^{2} \nabla_{r e l}^{2} / 2 \mu+\mu \omega^{2}\left(x^{2}+y^{2}\right) / 2+\mu\left(\omega_{z}^{2}+\omega_{c}^{2}\right) z^{2} / 2 \\
& +i \hbar \omega_{c} z \partial / \partial y+e^{2} / 4 \pi \epsilon \epsilon_{0} r,
\end{aligned}
$$

where $M=2 m$ and $\mu=m / 2$. The two-electron wave function can be written as a product of center-of-mass and relative eigenfunctions

$$
\Psi\left(\mathbf{r}_{1}, \mathbf{r}_{2}\right)=\Phi_{c m}\left[\left(\mathbf{r}_{1}+\mathbf{r}_{2}\right) / 2\right] \Phi_{\text {rel }}\left(\mathbf{r}_{1}-\mathbf{r}_{2}\right) .
$$

It can be verified by a direct calculation that the ground-state energy of the center-of-mass motion equals $E_{c m}=\hbar\left\{\omega+\left[\omega_{c}^{2}\right.\right.$ $\left.\left.+\left(\omega+\omega_{z}\right)^{2}\right]^{1 / 2}\right\} / 2$, and that the ground-state wave function reads (up to a normalization constant)

$$
\Phi_{c m}(\mathbf{R})=\exp \left(-b_{x} X^{2}-b_{y} Y^{2}-b_{z} Z^{2}+i b_{z y} Z Y\right),
$$

with $\quad b_{x}=M \omega / 2 \hbar, \quad b_{y}=M \omega \sqrt{1+\omega_{c}^{2} /\left(\omega_{z}+\omega\right)^{2}} / 2 \hbar, \quad b_{z}$ $=M \omega_{z} \sqrt{1+\omega_{c}^{2} /\left(\omega_{z}+\omega\right)^{2}} / 2 \hbar$, and $b_{z y}=M \omega_{c} \omega /\left(\omega_{z}+\omega\right) \hbar$.

The relative Hamiltonian (3) commutes with the parity operator $(\mathbf{r} \rightarrow-\mathbf{r})$. States with even (odd) parity are symmetric (antisymmetric) with respect to the interchange of electrons and therefore correspond to spin singlets (triplets). The relative Hamiltonian commutes also with operators of inversion in $x$ direction $(x \rightarrow-x)$ and in the plane perpendicular to the magnetic field $[(y, z) \rightarrow-(y, z)]$. In the following we will label the states by their parities in $x$ and $y$ directions put in parentheses by the first and second descriptor, respectively [for instance, (even,odd) stands for a state even in $x$ direction and odd in the $(y, z)$ plane].

In the absence of the electron-electron interaction the contribution of the diamagnetic $\left(m \omega_{c}^{2} z^{2} / 2\right)$ and the paramagnetic $\left(i \hbar \omega_{c} z \partial / \partial y\right)$ terms describing the in-plane magnetic field in Hamiltonians (1)-(3) can be evaluated analytically. For the center-of-mass Hamiltonian (2) and the wave function (5) the expectation values of the diamagnetic and paramagnetic terms equal $M \omega_{c}^{2} / 8 b_{z}=\hbar \omega_{c}^{2} / 4 \omega_{z} \sqrt{1+\omega_{c}^{2} /\left(\omega_{z}+\omega\right)^{2}}$ and $-\hbar \omega_{c} b_{z y} / 4 b_{z}=-\hbar \omega \omega_{c}^{2} / 2\left(\omega_{z}+\omega\right) \omega_{z} \sqrt{1+\omega_{c}^{2} /\left(\omega_{z}+\omega\right)^{2}}$, respectively. In the limit of infinite $\omega_{z}$ the electrons become confined two-dimensionally in the $z=0$ plane, which leads to the vanishing of the diamagnetic and paramagnetic contributions (it is also evident from the form of the corresponding terms in the Hamiltonian). In order to maintain the values of the
TABLE I. Convergence of the energy estimates obtained with wave function (6) to the exact ground-state energy (last row) of the relative Hamiltonian as function of $M$ (the number of terms used in the wave function is $K$ ) for $B=0$ and a spherically symmetric confinement potential with $\hbar \omega=\hbar \omega_{z}=3 \mathrm{meV}$.

\begin{tabular}{ccc}
\hline \hline$M$ & $K$ & $E(\mathrm{meV})$ \\
\hline 0 & 1 & 8.6100 \\
2 & 5 & 8.4336 \\
6 & 30 & 8.4187 \\
14 & 204 & 8.4145 \\
22 & 650 & 8.4134 \\
exact & & 8.4134 \\
\hline \hline
\end{tabular}

magnetic contributions to the energy, $\omega_{c}$ has to grow simultaneously with $\omega_{z}$. For the $\omega_{c} / \omega_{z}$ ratio kept constant the diamagnetic contribution is approximately linear in $\omega_{c}$ and the paramagnetic contribution is approximately constant.

In order to solve the eigenequation for the relative motion Hamiltonian in the presence of the interaction we use the variational method with the following trial wave function:

$$
\Phi_{r e l}(\mathbf{r})=\exp \left(-\alpha x^{2}-\beta y^{2}-\gamma z^{2}+i c z y\right) \sum_{i, j, k=0}^{i+j+k \leqslant M} d_{i j k} x^{i} y^{j} z^{k},
$$

where $\alpha, \beta, \gamma, c$ are the nonlinear parameters and $d_{i j k}$ is the linear variational parameter, $M$ controls the number of basis elements. In the absence of the interaction, the wave function (6) reproduces exactly the analytic eigenfunctions using a finite number of terms in the expansion. In this sense the present approach is equivalent to the three-dimensional generalization of the method used by Drouvelis et al. ${ }^{22,23}$ for two-dimensional anisotropic quantum dots with perpendicular magnetic field. Similar single-electron wave function was used by Harju et al. ${ }^{21}$ for the configuration-interaction study of the electron pair in two-dimensional laterally coupled quantum dots.

The exact wave function for the s-type states in a harmonic-oscillator confinement potential is asymptotically linear in the limit $r \rightarrow 0$ (has a cusp at $r=0$ ), which is related to the Coulomb interaction singularity. Since $r$ cannot be developed in a MacLaurin series in Cartesian coordinates the present and previously used wave functions ${ }^{21-23}$ cannot account for this linearity in the nearest neighborhood of the origin. We have performed test calculations in order to estimate the importance of this shortcoming. For this purpose we have solved the eigenequation for Hamiltonian (1) for $B=0$ and a spherically symmetric potential $\hbar \omega=\hbar \omega_{z}$ $=3 \mathrm{meV}$ in a numerically exact manner with a finite difference method. Table I shows the convergence of the energy estimates obtained with the wave function (6) to the exact ground-state eigenvalue. Comparison of the wave functions is presented in Fig. 1. For $M=6$ the wave function has a shallow local minimum at the origin and the maximum of the wave function is shifted to the right with respect to the exact solution. For $M=22$ the local minimum gets almost as deep 


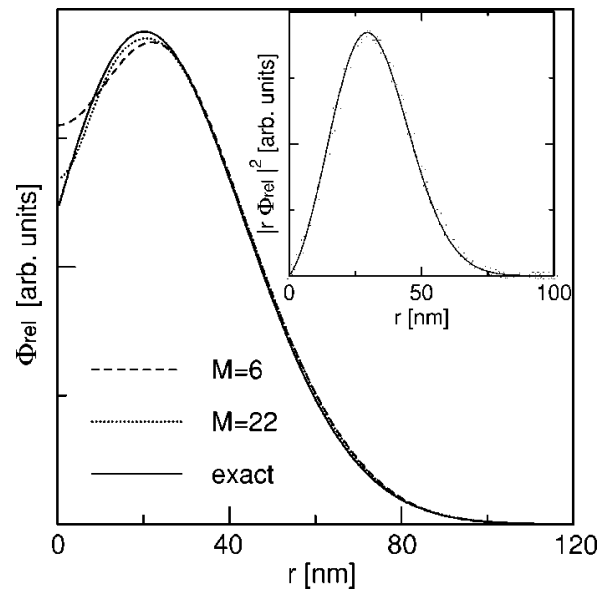

FIG. 1. Ground-state wave function of the relative Hamiltonian calculated for the parameters used in Table I. Solid line shows the exact wave function, the dashed (dotted) line is the wave function obtained by the variational method for $M=6(M=22)$. Inset: comparison of the exact radial probability density (solid line) with the dependence obtained variationally with $M=22$ (open circles).

as in the exact solution, the wave function becomes linear between 5 and $15 \mathrm{~nm}$, and the position of the maximum of the wave function is improved with respect to the $M=6$ wave function. The expectation value of the energy is a functional of the radial probability density, which is depicted in the inset of Fig. 1. We can see that the exact radial probability density and the one calculated for $M=22$ are indistinguishable, which explains the high precision of the energy estimates (cf. Table I). The region of the trial wave function nonlinearity around the origin can be made arbitrarily small, and a numerically exact value of the energy and a nearly exact radial probability density are obtained. Therefore the application of the proposed wave function is well justified. The cusp shortcoming concerns also the states of $p$ symmetry, but its importance is smaller since for $p$ states the functions vanish at the origin. Wave functions of higher angular momentum have no cusps at the origin. The cusps in the exact $s$ and $p$ symmetry states dissapear ${ }^{24}$ in the Wigner crystallization limit for which a Coulomb hole is formed in the relative wave function near $r=0$.

\section{RESULTS AND DISCUSSION}

\section{A. Spherical quantum dot}

We consider first the effect of the magnetic field on a spherical quantum dot. The lowest energy levels for all parity symmetries calculated with respect to the lowest Landau level are plotted in Fig. 2 as function of the external magnetic field. The solid (dotted) lines correspond to states of even (odd) total parity, i.e., to singlet (triplet) states. In the absence of a magnetic field the ground state corresponds to the angular momentum quantum number $L=0$, the lowest excited states of (even, odd) as well as with (odd, even) parity correspond to $L=1$. The lowest (odd, odd) energy level corresponds to $L=2$. The magnetic field breaks the spherical symmetry of the system so only the $x$-component

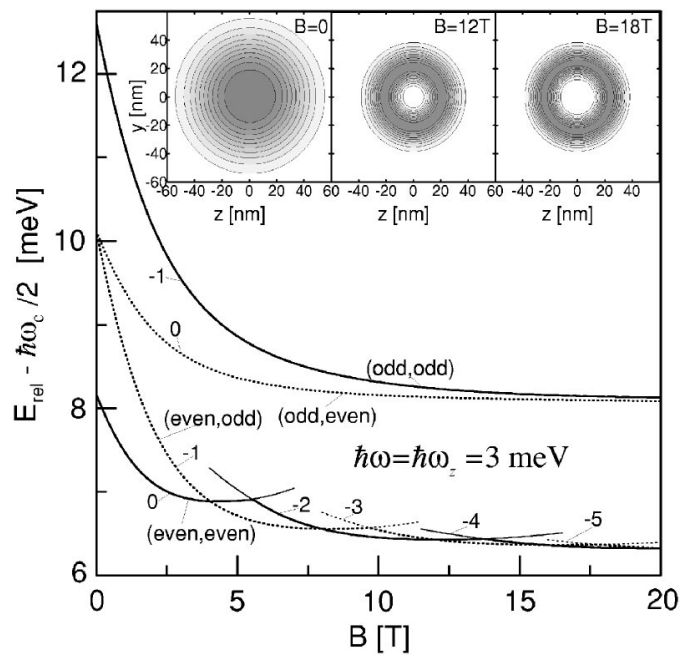

FIG. 2. Energy eigenvalues of the relative Hamiltonian (3) calculated with respect to the lowest Landau level for a spherical quantum dot with $\hbar \omega=\hbar \omega_{z}=3 \mathrm{meV}$ (Zeeman effect neglected). The solid and dotted lines show the singlet and triplet energy levels, respectively. In parentheses the parity of the corresponding eigenstates in the $x$ direction and within the $(y, z)$ plane is given. The numbers indicate the $x$ component of the angular momentum in $\hbar$ units. The inset shows the lowest singlet probability density integrated over the $x$ direction for magnetic fields $B=0,12$, and $18 \mathrm{~T}$.

of the angular momentum is quantized. Figure 2 shows that angular momentum and spin transitions appear in the ground state. The $x$ component of angular momentum of the lowest singlet (triplet) states takes values of even (odd) parity integers (in $\hbar$ units). The angular momentum transitions and the singlet-triplet oscillations are qualitatively similar to the effects appearing in two-dimensional circular quantum dots in the presence of a perpendicular magnetic field. ${ }^{23,25}$ The origin of the singlet-triplet oscillations in two-electron twodimensional quantum dots is well understood. The increase of the magnetic field pushes the maximum of the relative wave function toward the origin (in the three-dimensional case-towards the $x$ axis) increasing the mean value of the Coulomb interelectron repulsion. In consequence the state of a higher angular momentum (with stronger electron separation) acquires lower energy beyond some critical value of the magnetic field. The ground-state angular momentum (without the spin Zeeman term) takes the subsequent integer values as the magnetic field increases. The lowest-energy states of the odd (even) angular momentum are realized in the triplet (singlet) spin configuration, ${ }^{25}$ which leads to the singlettriplet oscillations.

On the other hand, the order of the lowest singlet and triplet energy levels that are odd in the $x$ direction is not affected by the field (cf. Fig. 2). Here, the magnetic-fieldinduced localization of the relative wave function around the $x$ axis does not essentially decrease the electron-electron distance since in these states the electrons are separated in the $x$ direction. The driving force for the singlet-triplet oscillations is therefore absent in this branch of energy levels.

The inset to Fig. 2 shows the contours of the relative probability density for the lowest singlet state integrated over the $x$ direction. Note that in the integration the minimum of 


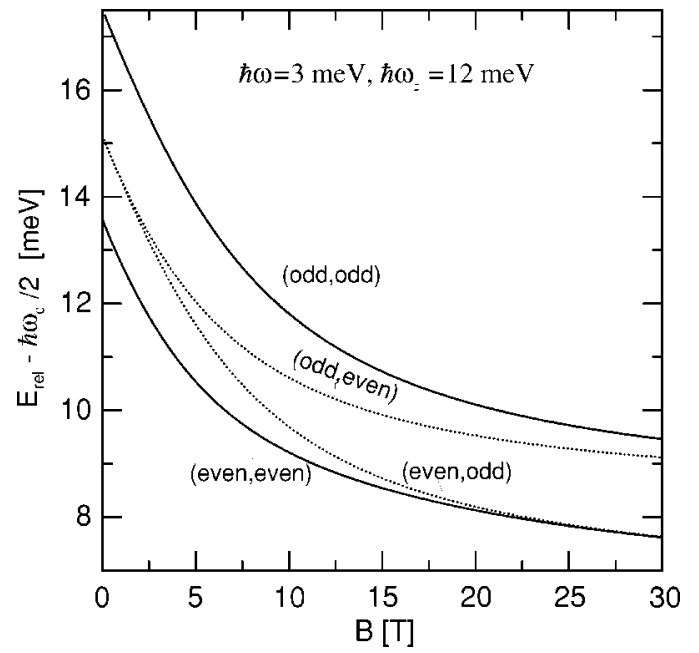

FIG. 3. Energy eigenvalues of the relative Hamiltonian calculated with respect to the lowest Landau level for a flat quantum dot with $\hbar \omega=3 \mathrm{meV}$ and $\hbar \omega_{z}=12 \mathrm{meV}$. The solid and dotted lines show the singlet and triplet energy levels, respectively. In parentheses the parity of the corresponding eigenstates in the $x$ direction and within the $(y, z)$ plane is given.

the probability density at the origin for $B=0$ (cf. Fig. 1) disappears. The lowest singlet states for $B=0,12$, and $18 \mathrm{~T}$ correspond to the $x$ component of the angular momentum equal to $0,-2 \hbar$, and $-4 \hbar$, respectively. For nonzero angular momentum, the probability density is totally removed from the $x$ axis. The densities exhibit perfect circular symmetry around the $x$ axis, although it is not evident from the form of the Landau-gauge Hamiltonian [cf. Eq. (3)]. This result can serve as an additional test of the reliability of the numerical approach applied.

\section{B. Flat quantum dot}

The low-energy spectrum for a flat quantum dot with $\hbar \omega=3 \mathrm{meV}$ and $\hbar \omega_{z}=12 \mathrm{meV}$ is plotted in Fig. 3 (Zeeman effect neglected). For this value of the $z$ confinement energy the spread of the electron charge density in the $z$ direction $2 \Delta z=2\left\langle z^{2}\right\rangle^{1 / 2}=\sqrt{2 \hbar / m \omega_{z}}$ is about $13.75 \mathrm{~nm}$. The magnetic field lifts the degeneracy of the triplet energy levels, which for $B=0$ correspond to states with $z$-component angular momentum equal to $\pm \hbar$. In the presence of a magnetic field oriented along the $x$ direction none of the components of the angular momentum commute with the Hamiltonian and therefore none of them are quantized. The external magnetic field leads to singlet-triplet degeneracy in contrast to singlettriplet oscillations observed in a spherical dot (cf. Fig. 2). Figures 2 and 3 show that the lowest singlet as well as the lowest triplet states have even $x$ direction parity, independently of the value of the magnetic field and the strength of the electron-electron interaction.

The singlet-triplet energy splitting in the absence of the spin Zeeman effect is shown in more detail in Fig. 4. The exchange energy for the spherical quantum dot has a discontinuous derivative when angular momentum transitions appear in the lowest singlet or triplet states. For small aniso-

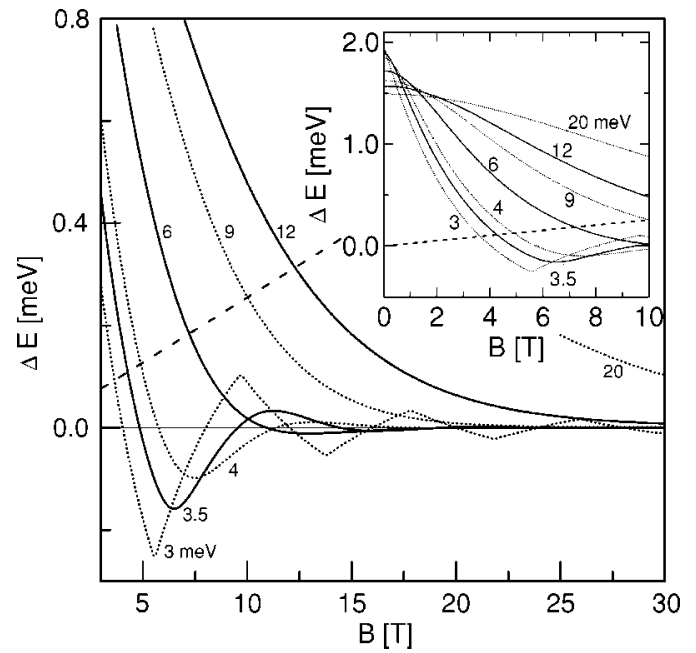

FIG. 4. Triplet-singlet energy difference (without the spin Zeeman effect) as function of the magnetic field for $\hbar \omega=3 \mathrm{meV}$ and various confinement energies in the $z$ direction. The thick dashed line shows the spin Zeeman splitting between the triplet and the singlet states. The inset shows the low-magnetic-field region.

tropy of the confinement potential (cf. the line for $\hbar \omega_{z}$ $=3.5 \mathrm{meV}$ ), the exchange energy becomes a smooth function of the magnetic field. The oscillations of the energy difference around zero have a decreasing amplitude with growing confinement energy in the $z$ direction. For $\hbar \omega_{z} \geqslant 8 \mathrm{meV}$ the oscillations disappear and the exchange energy decreases monotonically to zero with the external field. The thick dashed line in Fig. 4 shows the spin Zeeman splitting between the singlet and triplet states $\left(-B \hbar g^{*} \mu_{B}\right.$ with the effective Landé factor $\left.g^{*}=-0.44\right)$. The crossing of this line with the exchange energy curves indicate the value of the magnetic field for which the triplet becomes the ground state.

The inset to Fig. 4 shows the exchange energy in the low magnetic field region. For $B=0 \mathrm{~T}$ the singlet-triplet energy splitting is a decreasing function of the strength of confinement in the $z$ direction. As the $z$ size of the quantum dot is decreased the system starts to approach the strictly twodimensional limit in which the singlet-triplet splitting for $\hbar \omega=3 \mathrm{meV}$ is $1.3 \mathrm{meV}$. The increase of the $z$ confinement energy affects more strongly the energy of the singlet state, increasing the value of the wave function at the origin (corresponding to both electrons localized in the same position). The triplet wave function vanishes at the origin due to the Pauli exclusion principle.

Let us now look at the origin behind the magnetic-fieldinduced singlet-triplet degeneracy for the dot with $\hbar \omega_{z}$ $=12 \mathrm{meV}$. The contour plots in Fig. 5 show the relative probability density integrated over $x$ (left panel) and $z$ (right panel) coordinates for the lowest singlet state. The quasithree-dimensional plots at the right-hand side of Fig. 5 show the surface in the $z>0$ half space at which the probability density falls to one-fifth of its maximum value. Region inside the surface contains roughly $90 \%$ of the probability. For $B=0 \mathrm{~T}$ the probability density integrated over the $x$ direction exhibits local maxima outside the $y=0$ line (cf. left panel of the contour plot in Fig. 5). The magnetic field transforms them into separated islands on the $(y, z)$ plane. For $B=0 \mathrm{~T}$ 


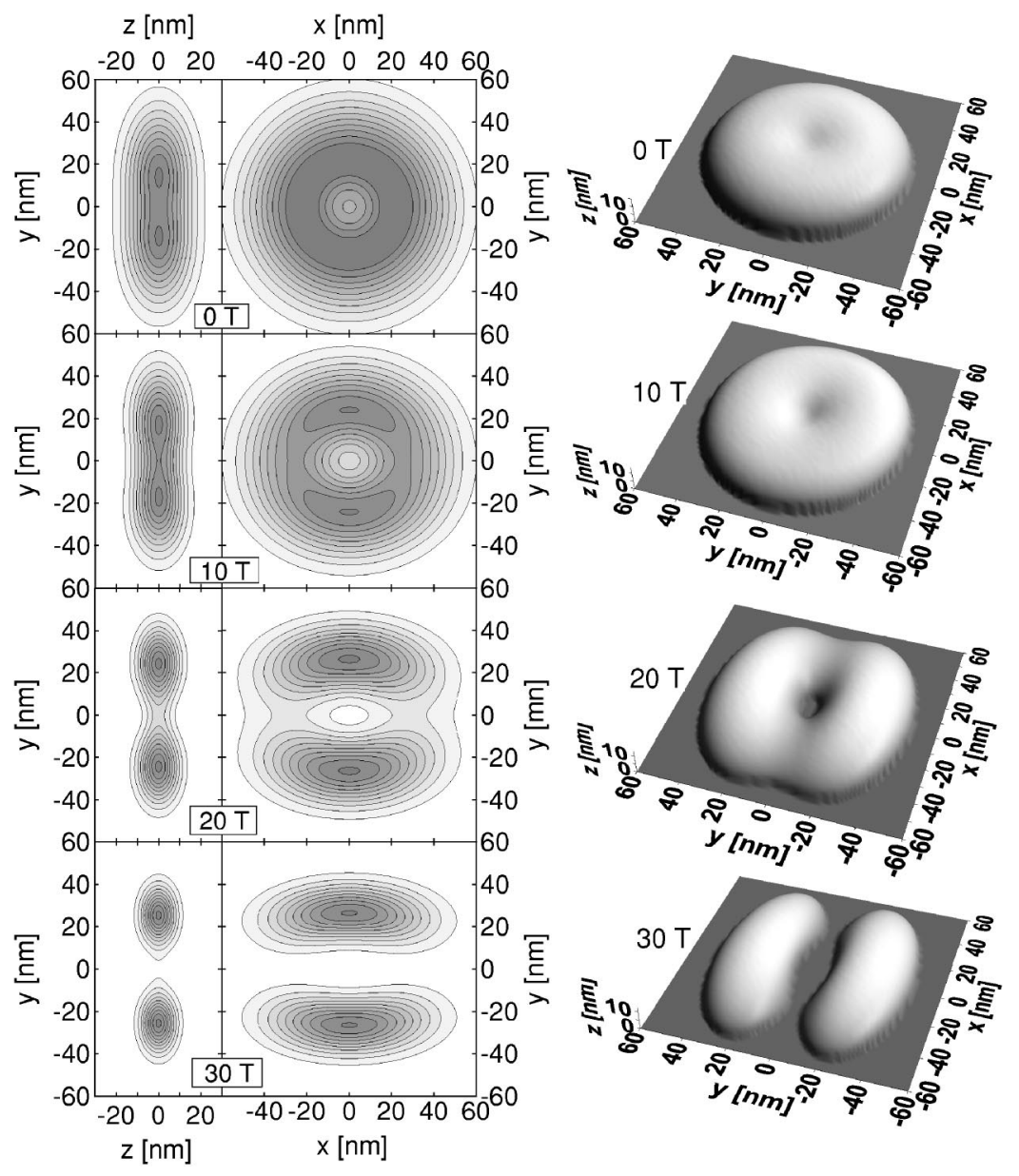

FIG. 5. Contour plots at the left side of the figure shows the relative motion probability density for the lowest singlet state integrated over the $x$ direction (left panel) and over the $z$ coordinate (right panel) for $\hbar \omega=3 \mathrm{meV}, \hbar \omega_{z}=12 \mathrm{meV}$, and different magnetic fields. Larger values of density are marked with darker colors. At the right side of the figure we show the surface at which the probability density takes one-fifth of its maximum value.

the density integrated over the $z$ coordinate is circular symmetric (cf. right panel of the contour plot in Fig. 5). For the flat quantum dot the integration over the $z$ coordinate does not fill in the central local maximum as in the spherical case (cf. Fig. 1 and inset of Fig. 2 for $B=0$ T). When a magnetic field is applied, the density loses its circular symmetry and is transformed into two maxima elongated along the direction of the magnetic field $(x)$ and becomes strongly localized in the direction perpendicular to the field. The region in which the probability density is nonzero at high magnetic field resembles two beans put along the $x$ axis near the $z=0$ plane.

Figure 6 shows the relative probability density for the triplet state [of odd parity in the $(y, z)$ plane and of even parity in the $x$ direction]. For $B=0$ the $y$ and $z$ degrees of freedom are decoupled and the wave function is simply of odd parity in the $y$ direction and even in $z$ direction. ${ }^{26}$ The plots for $B=0 \mathrm{~T}$ show that the density vanishes near the $y$ $=0$ plane and that it forms two semiround islands parallel to the $x$ axis localized around the $z=0$ plane. Magnetic field makes the islands thinner, longer, and less oval. For $B$ $=30 \mathrm{~T}$ the probability densities for the triplet and singlet states become almost identical, which is the reason for the singlet-triplet degeneracy at high magnetic field (cf. Fig. 3).

For the sake of physical interpretation it is useful to look at the ground-state charge density in the laboratory frame of reference. The charge density can be extracted from the twoelectron wave function by integration with Dirac $\delta$ functions

$$
\begin{aligned}
n(\mathbf{r}) & =\left\langle\Psi\left(\mathbf{r}_{1}, \mathbf{r}_{2}\right)\left|\delta\left(\mathbf{r}-\mathbf{r}_{1}\right)+\delta\left(\mathbf{r}-\mathbf{r}_{2}\right)\right| \Psi\left(\mathbf{r}_{1}, \mathbf{r}_{2}\right)\right\rangle \\
& =2 \int d \mathbf{r}_{1}\left|\Phi_{c m}\left(\left(\mathbf{r}_{1}+\mathbf{r}\right) / 2\right)\right|^{2}\left|\Phi_{r e l}\left(\mathbf{r}_{1}-\mathbf{r}\right)\right|^{2} .
\end{aligned}
$$

Figure 7 shows the comparison of the probability densities of the center-of-mass and relative ground states and the twoelectron charge densities (divided for comparison by two) integrated over the $z$ direction for the potential parameters of Figs. 5 and $6 .{ }^{27}$ The noninteracting density (divided by two) and the center-of-mass density come from solutions of the same Schrödinger equations, the only difference is that the center of mass is twice heavier, which results in a stronger localization of the center-of-mass density. On the other hand, the mass that enters the relative Hamiltonian is half of the electron mass, which along with the repulsive Coulomb potential results in a weaker localization of the relative charge density. The interacting two-electron charge density calculated according to expression (7) is more weakly localized than the center-of-mass density but the localization is stronger than for the relative wave function. The integration of the relative charge density with the center-of-mass density fills the Coulomb hole visible in the relative density (cf. right panel of Fig. 5 for $B=0 \mathrm{~T}$ ). The effect of the Coulomb interaction on the electron localization can be estimated from a comparison of the interacting and noninteracting charge densities. 


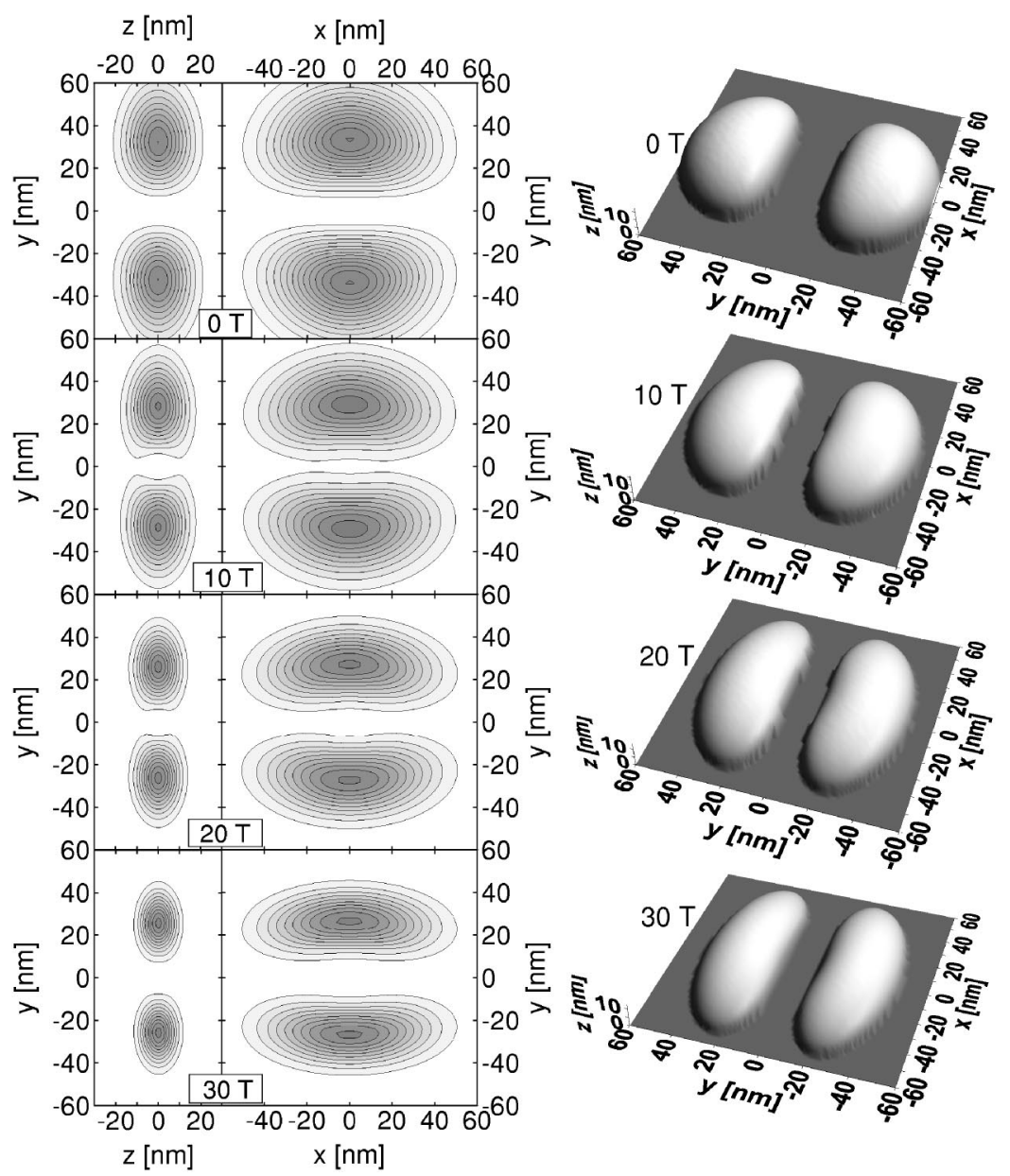

FIG. 6. The same as Fig. 5 but now for the lowest triplet state.

The evolution of the ground-state charge density $[n(\mathbf{r})]$ with the in-plane magnetic field, which is presented in Fig. 8. The two local maxima of the probability density visible for the relative eigenstate (cf. left plot for $B=0 \mathrm{~T}$ in Fig. 5)

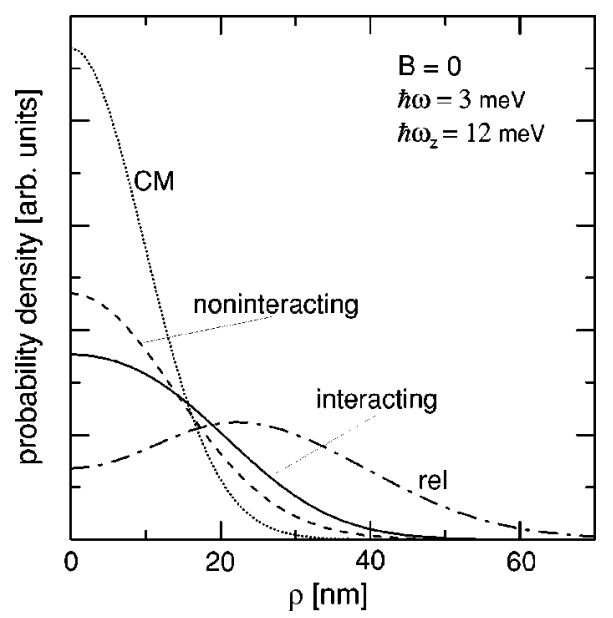

FIG. 7. Probability densities of the ground state of the center of mass (CM) and relative (rel) Hamiltonians, and the charge density of the noninteracting and interacting system of two electrons integrated over the $z$ direction as a function of the radial coordinate $\rho$ $=\sqrt{x^{2}+y^{2}}$ for $\hbar \omega=3 \mathrm{meV}, \hbar \omega_{z}=12 \mathrm{meV}$, and $B=0 \mathrm{~T}$. The twoelectron charge densities have been divided by 2 . merge into a central maximum when integrated over the relative probability density with the center-of-mass density. For $B=10 \mathrm{~T}$ the limits of the charge pool become squeezed in the direction perpendicular to the field. The appearance of the maximum elongated along the $x=0$ axis (cf. right plot for $B=10 \mathrm{~T}$ ) is due to the formation of the two maxima in the relative density (cf. right plot in Fig. 5 for $B=10 \mathrm{~T}$ ). Plots for $B=20 \mathrm{~T}$ and $B=30 \mathrm{~T}$ show a distinct separation of the electron charges, i. e., Wigner crystallization. The single-electron charge islands formed under the influence of the in-plane field form stripes elongated along the direction of the applied field. The extent of the charge density is not essentially changed along the direction of the field.

The magnetic-field-induced singlet-triplet degeneracy can be conveniently explained in the single-electron picture as due to the vanishing overlap between the wave functions of the two electrons. In the absence of the overlap the exchange interaction disappears leading to the observed spin degeneracy. The wave function separation is due to the strong localization of the single-electron charge islands in the direction perpendicular to the field. The present effect is similar to the singlet-triplet degeneracy induced by the in-plane magnetic field for laterally coupled quantum dots. ${ }^{19,20}$ For coupled dots the in-plane magnetic field induces stronger localization in each of the quantum dots, which results in a decreasing tunnel coupling (an increase of the effective height of the interdot barrier), which eventually leads to the 


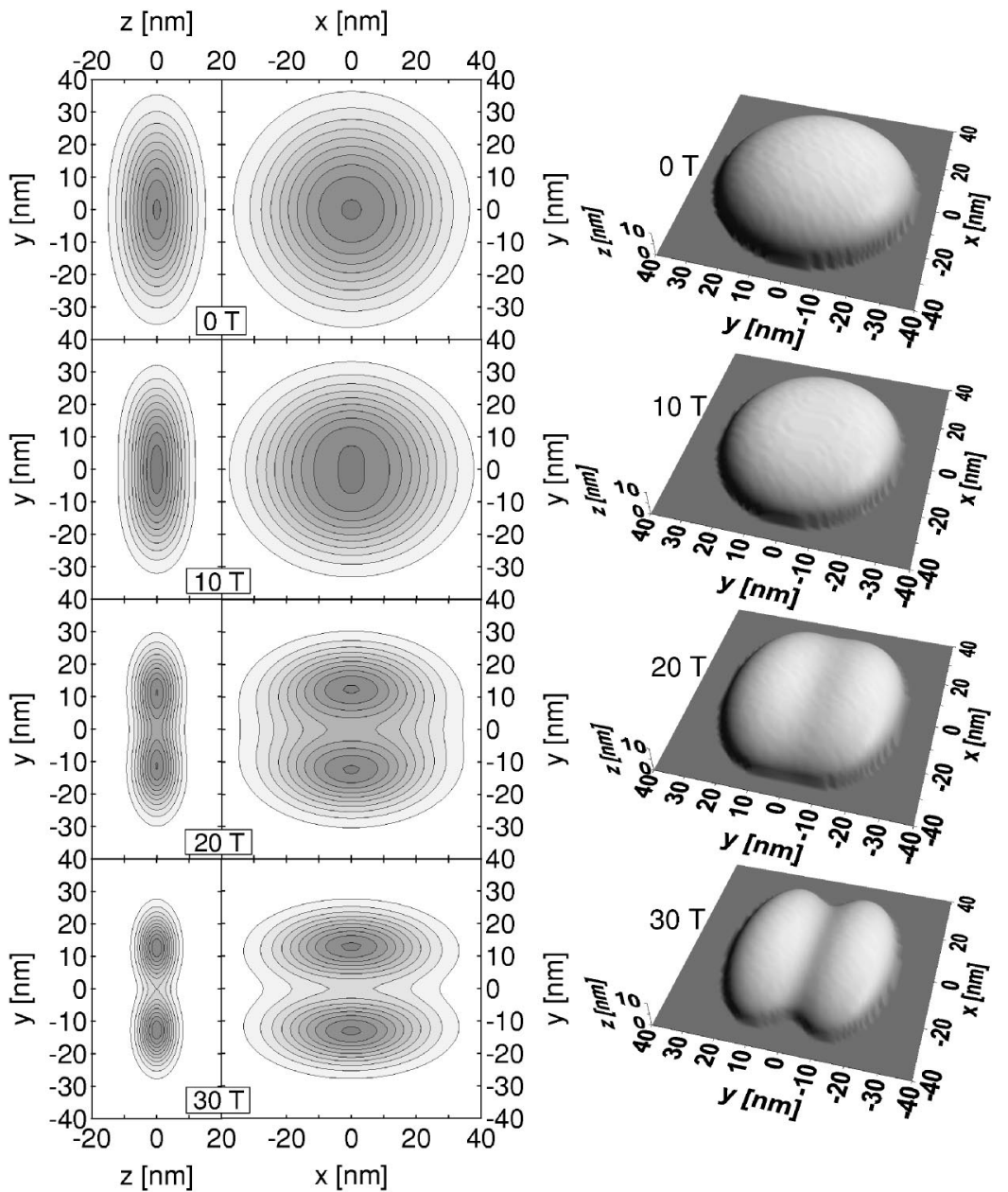

FIG. 8. Contour plots at the left side of the figure show the two-electron probability densities $[n(\mathbf{r})]$ integrated over the $x$ (left panel) and $z$ coordinates (right panel) for $\hbar \omega=3 \mathrm{meV}, \hbar \omega_{z}$ $=12 \mathrm{meV}$. The surface plots at the right side of the figure show the surface at which the charge density takes one-fifth of its maximum value. separation of the single-electron wave functions. The separation accompanied with the singlet-triplet degeneracy appears also without the in-plane field for the thick interdot barrier ${ }^{28}$ as well as in large quasi-one-dimensional quantum dots. ${ }^{28,29}$ Drouvelis et al. $^{23}$ found the ground-state singlet-triplet degeneracy for large anisotropy in a small two-dimensional quantum dot in the absence of the external magnetic field. For strong anisotropy this model ${ }^{23}$ gives a strictly onedimensional potential, for which the Coulomb interaction is extremely strong, leading to Wigner crystallization even for dots of relatively small size.

Since the laboratory frame separation is accompanied by a singlet-triplet degeneracy one can use the vanishing value of the exchange energy to propose a criterium for the magneticfield-induced Wigner crystallization. Figure 9 shows the triplet-singlet energy difference (Zeeman effect neglected) on the $\left(\hbar \omega_{z}, B\right)$ plane for $\hbar \omega=3 \mathrm{meV}$. The magnetic field for which the exchange energy becomes negligible is a distinctly growing function of $\hbar \omega_{z}$. The magnetic field above which the exchange energy falls below $0.1 \mathrm{meV}$ for $\hbar \omega_{z}>4 \mathrm{meV}$ can be very well approximated by a linear dependence $B$ $=1.6\left(\hbar \omega_{z}-1.266 \mathrm{meV}\right) \mathrm{T} / \mathrm{meV}$. The value of the magneticfield-inducing Wigner crystallization is an increasing function of $\hbar \omega$-the confinement energy in the $(x, y)$ plane. For $\hbar \omega_{z}>\frac{4}{3} \hbar \omega$ the exchange energy falls to $0.1 \mathrm{meV}$ at $B$ $=1.24\left(\hbar \omega_{z}-0.37 \mathrm{meV}\right) \mathrm{T} / \mathrm{meV}, \quad B=2.1\left(\hbar \omega_{z}-1.72 \mathrm{meV}\right)$

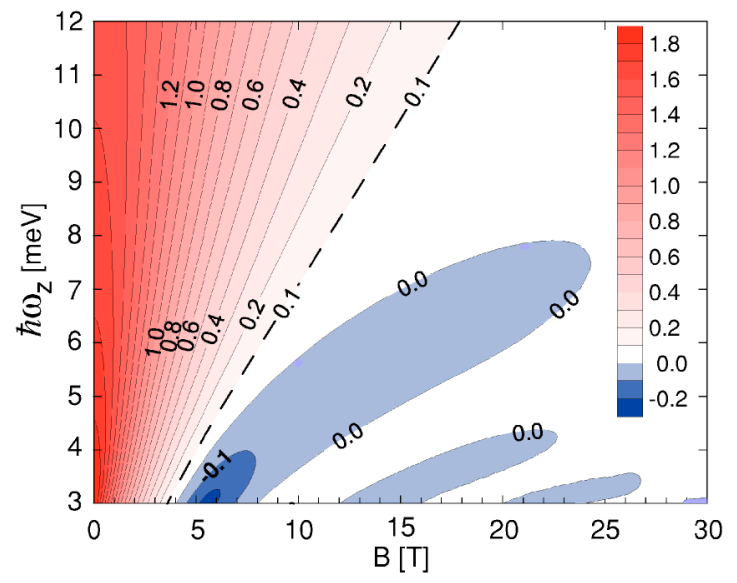

FIG. 9. (Color online) Triplet-singlet energy difference as a function of the magnetic field and vertical confinement energy for $\hbar \omega=3 \mathrm{meV}$. Blue (white and red) regions correspond to the triplet (singlet) ground state for the spin Zeeman effect neglected. The dashed line shows the values of $B$ and $\hbar \omega_{z}$ for which the tripletsinglet energy difference is equal to $0.1 \mathrm{meV}$. Color scale is given at the right-hand side of the figure. 
$\mathrm{T} / \mathrm{meV}, B=2.95\left(\hbar \omega_{z}-3.74 \mathrm{meV}\right) \mathrm{T} / \mathrm{meV}$ for $\hbar \omega=2,5$, and

$10 \mathrm{meV}$, respectively.

In spherical quantum dots (cf. Fig. 2) as well as in circular two-dimensional quantum dots in a perpendicular magnetic field $^{23,25}$ singlet-triplet oscillations are observed instead of the above degeneracy. But in these systems the separation of the electron charges appears in the inner degrees of freedom not in the laboratory frame, so that the picture of vanishing overlap between the single-electron wave functions does not apply (it would imply breaking of the symmetry of the external potential).

In the present paper we have used a harmonic confinement potential in the growth direction. The potential in real dots has often a quantum-well or a triangular form. Although the shape of the confinement in the growth direction should not have a qualitative influence on the results, one should expect quantitative differences except in the region where the magnetic field is so strong that the magnetic length $(\sqrt{2 \hbar / e B}=36.28 / \sqrt{B} \mathrm{~nm} \sqrt{T})$ is much smaller than the range of the vertical confinement. In quantum wells the energy spacings between the lowest-energy levels are larger than that for the harmonic-confinement potential. The spacings between the lowest-energy levels for an infinite quantum well with width $13.75 \mathrm{~nm}$ (corresponding to a similar vertical spread of the electron wave function for $\hbar \omega_{z}=12 \mathrm{meV}$ ) equal $22 \mathrm{meV}$. Therefore, the in-plane magnetic field values inducing Wigner crystallization in dots with well-like vertical confinement will be larger than the ones found in this paper for the harmonic-confinement potential.

\section{SUMMARY AND CONCLUSIONS}

We have studied the orbital effects due to the external magnetic field oriented along the $x$ axis on two electrons confined within a harmonic three-dimensional quantum dot rotationally symmetric with respect to the $z$ axis. Calculations used explicitly the center-of-mass separation and were performed with Gaussian trial wave functions. In flat quantum dots a high in-plane magnetic field leads eventually to spin degeneracy (in the absence of the spin Zeeman interaction) instead of spin-triplet oscillations, which are obtained for magnetic field oriented along the axis of a cylindrical symmetric quantum dot. The spin degeneracy is due to Wigner crystallization induced in the laboratory frame by the in-plane magnetic field. For flat quantum dots and low magnetic fields the orbital effects have initially a negligible effect on the singlet-triplet energy splitting. In the high-magneticfield limit, when Wigner molecules are formed, all the singlet-triplet splitting can be strictly attributed to spinrelated effects. Nevertheless, between the low-field and molecular regimes there exists a magnetic field interval for which the exchange energy rapidly changes with the magnetic field.

\section{ACKNOWLEDGMENTS}

This paper was partly supported by the Polish Ministry of Scientific Research and Information Technology in the framework of the solicited grant PBZ-MIN-008/P03/2003, the Flemish Science Foundation (FWO-Vl), the Belgian Science Policy, and the University of Antwerpen (VIS and GOA). The first author is supported by the Foundation for Polish Science (FNP) and by Marie Curie IEF Project No. MEIF-CT-2004-500157.
*Electronic address: francois.peeters@ua.ac.be

${ }^{1}$ L. Jacak, P. Hawrylak, and A. Wójs, Quantum Dots (Springer Berlin, 1998).

${ }^{2}$ S. M. Reimann and M. Manninen, Rev. Mod. Phys. 74, 1283 (2002).

${ }^{3}$ L. P. Kouwenhoven, D. G. Austing, and S. Tarucha, Rep. Prog. Phys. 64, 701 (2001).

${ }^{4}$ V. M. Bedanov and F. M. Peeters, Phys. Rev. B 49, 2667 (1994).

${ }^{5}$ B. Szafran, F. M. Peeters, S. Bednarek, and J. Adamowski, Phys. Rev. B 69, 125344 (2004).

${ }^{6}$ M. Manninen, M. Koskinen, S. M. Reimann, and B. Mottelson, Eur. Phys. J. D 16, 381 (2001).

${ }^{7}$ B. Reusch and R. Egger, Europhys. Lett. 64, 84 (2003).

${ }^{8}$ A. D. Güçlü, J.-S. Wang, and H. Guo, Phys. Rev. B 68, 035304 (2003)

${ }^{9}$ B. Szafran and F. M. Peeters, Europhys. Lett. 66, 701 (2004).

${ }^{10}$ J. A. Folk, S. R. Patel, K. M. Birnbaum, C. M. Marcus, C. I. Duruöz, and J. S. Harris Jr., Phys. Rev. Lett. 86, 2102 (2001).

${ }^{11}$ R. Hanson, B. Witkamp, L. M. Vandersypen, L. H. Willems van Beveren, J. M. Elzerman, and L. P. Kouwenhoven, Phys. Rev. Lett. 91, 196802 (2003).

${ }^{12}$ R. M. Potok, J. A. Folk, C. M. Marcus, V. Umansky, M. Hanson, and A. C. Gossard, Phys. Rev. Lett. 91, 016802 (2003).

${ }^{13}$ B. I. Halperin, A. Stern, Y. Oreg, J. N. H. J Cremers, J. A. Folk, and C. M. Marcus, Phys. Rev. Lett. 86, 2106 (2001).

${ }^{14}$ M. Valín-Rodriguez, A. Puente, and L. Serra, Phys. Rev. B 69, 085306 (2004).

${ }^{15}$ S. Tarucha, D. G. Austing, T. Honda, R. J. van der Hage, and L. P. Kouwenhoven, Phys. Rev. Lett. 77, 3613 (1996).

${ }^{16}$ R. C. Ashoori, H. L. Stormer, J. S. Weiner, L. N. Pfeiffer, S. J. Pearton, K. W. Baldwin, and K. W. West, Phys. Rev. Lett. 68, 3088 (1992).

${ }^{17}$ J. Schmid, J. Weis, K. Eberl, and K. v. Klitzing, Phys. Rev. Lett. 84, 5824 (2000).

${ }^{18}$ F. Stern and S. Das Sarma, Phys. Rev. B 30, 840 (1984).

${ }^{19}$ D. Bellucci, M. Rontani, F. Troiani, G. Goldoni, and E. Molinari, Phys. Rev. B 69, 201308 (2004).

${ }^{20}$ G. Burkard, D. Loss, and D. P. Di Vicenzo, Phys. Rev. B 59, 2070 (1999).

${ }^{21}$ A. Harju, S. Siljamäki, and R. M. Nieminen, Phys. Rev. Lett. 88, 226804 (2002).

${ }^{22}$ P. S. Drouvelis, P. Schmelcher, and F. K. Diakonos, Europhys. Lett. 64, 232 (2003).

${ }^{23}$ P. S. Drouvelis, P. Schmelcher, and F. K. Diakonos, Phys. Rev. B 
69, 155312 (2004); 69, 035333 (2004).

${ }^{24}$ B. Szafran, J. Adamowski, and S. Bednarek, Physica E (Amsterdam) 5, 185 (2000).

${ }^{25}$ M. Dineykhan and R. G. Nazmitdinov, J. Phys.: Condens. Matter 11, L83 (1999).

${ }^{26}$ This wave function is not the eigenfunction of the $z$ component angular momentum. However, it can be constructed from a su- perposition of degenerate wave functions with the $z$-component angular momentum $\pm \hbar$ at $B=0$.

${ }^{27}$ The charge densities are normalized with the Jacobian $\rho$.

${ }^{28}$ S. Bednarek, T. Chwiej, J. Adamowski, and B. Szafran, Phys. Rev. B 67, 205316 (2003).

${ }^{29}$ B. Szafran, F. M. Peeters, S. Bednarek, T. Chwiej, and J. Adamowski, Phys. Rev. B 70, 035401(2004). 